\title{
On the Glicksberg theorem for locally quasi-convex Schwartz groups
}

by

\author{
Lydia Außenhofer (Eichstätt)
}

\begin{abstract}
We prove that every locally quasi-convex Schwartz group satisfies the Glicksberg theorem for weakly compact sets.
\end{abstract}

1. Introduction. For an abelian topological group $(G, \tau)$ the group of continuous homomorphisms $G \rightarrow \mathbb{T}$, where $\mathbb{T}=\mathbb{R} / \mathbb{Z}$, is denoted by $G^{\wedge}$ and called the character group. $G^{\wedge}$ is endowed with the compact-open topology. The elements of $G^{\wedge}$ are named continuous characters. The topology on $G$ induced by all continuous characters, i.e. by the mapping $G \rightarrow \mathbb{T}^{G^{\wedge}}, x \mapsto$ $(\chi(x))_{\chi \in G^{\wedge}}$, is called the weak topology. The group $G$ endowed with the weak topology will be denoted by $G^{+}$.

The famous Glicksberg theorem states that for a locally compact abelian (LCA for short) group $G$ the compact subsets of $G$ and $G^{+}$coincide.

For discrete groups this was shown by Leptin $([22])$ and in full generality by Glicksberg ([16]). Since then it has been generalized in various manners:

- To non-abelian groups: [15], [26], and [30];

- To $\operatorname{Hom}(G, H)$ where $G$ and $H$ are topological groups: [13] and [29];

- By other characterizations and generalizations: [11], [12], [14], [19], and $[20]$;

- By studying properties of the weak topology: [18], [27], [28], and [30];

- To larger classes of groups (beyond LCA): [5], [6], and [25].

In this paper we take up the last point of view and we generalize the Glicksberg theorem to the class of locally quasi-convex Schwartz groups introduced in [3], a class of groups which contains all nuclear groups, all locally convex Schwartz vector spaces and all locally quasi-convex groups which are

2000 Mathematics Subject Classification: 22A05, 46A11.

Key words and phrases: Glicksberg's theorem, Bohr topology, Schwartz groups, locally quasi-convex group, Namioka's theorem, $k$-space. 
hemicompact $k$-spaces, in particular, the character groups of abelian metrizable groups.

The paper is structured as follows: In the second section we gather auxiliary results, most of them well known. We have included the elementary proofs of some of these in order to make the paper self-contained. In the third section we introduce qc-precompact sets, a class of subsets which contains all precompact sets, and prove that in locally quasi-convex groups both classes coincide.

In the fourth section we prove the Glicksberg theorem for locally quasiconvex Schwartz groups. As a first step we show that every weakly compact set in a locally quasi-convex group has a property which is slightly weaker than being bounded. In the theory of locally convex vector spaces the fact that weakly bounded sets are bounded in the original topology can be proved by applying the principle of uniform boundedness. Here we replace this theorem by Namioka's theorem on joint continuity in order to obtain a similar result in the group case. Combining this with the definition of a Schwartz group and the result on qc-precompact sets established before, we finally deduce that in a locally quasi-convex Schwartz group $G$ every weakly compact set is compact. In the last section this result is applied to the character groups of metrizable groups which form an example of Schwartz groups.

2. Background and notation. In this section we gather some facts which will be used in the following sections.

2.1. Weakly continuous homomorphisms. The set of all neighbourhoods of the neutral element 0 of an abelian topological group $(G, \tau)$ will be denoted by $\mathcal{U}_{\tau}(0)$ or, if no confusion can arise, by $\mathcal{U}(0)$.

Let $(X, \mathcal{O})$ be a topological space. For a subset $X_{0} \subseteq X$ we denote by $\left(X_{0},\left.\mathcal{O}\right|_{X_{0}}\right)$ the induced topology.

We identify $\mathbb{T}$ with $]-1 / 2,1 / 2]$ and define $\mathbb{T}_{+}:=[-1 / 4,1 / 4]$.

Proposition 2.1.

(i) Let $\phi: H \rightarrow G$ be a continuous homomorphism between abelian Hausdorff groups. Then $\phi: H^{+} \rightarrow G^{+}$is also continuous.

(ii) If $H$ is a dually embedded subgroup of $G$ then $H^{+}$is topologically isomorphic to a subgroup of $G^{+}$. (Recall that a subgroup is called dually embedded if every continuous character of the subgroup can be extended to a continuous character of the whole group.)

Proof. (i) Since the weak topology on $G$ is induced by all continuous characters, it is sufficient to prove that $H^{+} \rightarrow \mathbb{T}, x \mapsto \chi(\phi(x))$, is continuous for every $\chi \in G^{\wedge}$. But this is clear since $\chi \circ \phi: H \rightarrow \mathbb{T}$ is continuous and hence $\chi \circ \phi \in H^{\wedge}$. 
(ii) Consider the embedding $\iota: H \rightarrow G$. According to (i), $\iota: H^{+} \rightarrow G^{+}$ is a continuous monomorphism. Let $\chi_{1}, \ldots, \chi_{n} \in H^{\wedge}$. By assumption, these continuous characters can be extended to $\psi_{1}, \ldots, \psi_{n} \in G^{\wedge}$. As $\iota\left(\bigcap_{j=1}^{n}\{x \in H\right.$ : $\left.\left.\chi_{j}(x) \in \mathbb{T}_{+}\right\}\right)=\bigcap_{j=1}^{n}\left\{x \in G: \psi_{j}(x) \in \mathbb{T}_{+}\right\} \cap \iota(H)$, we conclude that $H^{+}$is embedded in $G^{+}$.

2.2. Polars and quasi-convex sets. For a subset $A$ of an abelian topological group $(G, \tau)$, the set $A^{\triangleright}=\left\{\chi \in G^{\wedge}: \chi(A) \subseteq \mathbb{T}_{+}\right\}$is called the polar of $A$, and for a subset $D \subseteq G^{\wedge}$, the set $D^{\triangleleft}:=\left\{x \in G: \chi(x) \in \mathbb{T}_{+} \forall \chi \in D\right\}$ is called the inverse polar of $D$. Of course,

$$
\begin{aligned}
& A \subseteq B \subseteq G \quad \Rightarrow B^{\triangleright} \subseteq A^{\triangleright} \subseteq G^{\wedge}, \\
& C \subseteq D \subseteq G^{\wedge} \Rightarrow D^{\triangleleft} \subseteq C^{\triangleleft} \subseteq G .
\end{aligned}
$$

A set $\emptyset \neq A \subseteq G$ is called quasi-convex if for every $x \in G \backslash A$ there exists $\chi \in A^{\triangleright}$ such that $\chi(x) \notin \mathbb{T}_{+}$. Hence $A$ is quasi-convex iff $A=\left(A^{\triangleright}\right)^{\triangleleft}$. E.g. $[-1 / 4 n, 1 / 4 n]$ is a quasi-convex subset of $\mathbb{T}$. A subset $A$ of an abelian group $G$ is called algebraically quasi-convex if it is quasi-convex when $G$ is endowed with the discrete topology.

LEMMA 2.2 .

(i) Intersections of quasi-convex sets are quasi-convex.

(ii) Inverse images of quasi-convex sets under continuous homomorphisms are quasi-convex.

(iii) Polars and inverse polars are quasi-convex.

Proof. (i) and (ii) are straightforward.

(iii) Fix $A \subseteq G$ and let $\chi \notin A^{\triangleright}$. Hence there exists $a \in A$ such that $\chi(a) \notin \mathbb{T}_{+}$. The mapping $\alpha_{G}(a): G^{\wedge} \rightarrow \mathbb{T}, \chi \mapsto \chi(a)$, is continuous, satisfies $\alpha_{G}(a)\left(A^{\triangleright}\right) \subseteq \mathbb{T}_{+}$and $\alpha_{G}(a)(\chi)=\chi(a) \notin \mathbb{T}_{+}$.

The proof that $C^{\triangleleft}$ is quasi-convex for every subset $C \subseteq G^{\wedge}$ is even easier.

According to 2.2(i), for a non-empty set $B \subseteq G$, the smallest quasiconvex set which contains $B$ exists. It is called the quasi-convex hull and will be denoted by qc $(B)$. We have

$$
\mathrm{qc}(B)=\left(B^{\triangleright}\right)^{\triangleleft} .
$$

This can be proved as follows: Since $B \subseteq\left(B^{\triangleright}\right)^{\triangleleft}$ and the set on the right hand side is quasi-convex $(2.2($ iii $))$, we obtain $\mathrm{qc}(B) \subseteq\left(B^{\triangleright}\right)^{\triangleleft}$. Conversely, let $Q \supseteq B$ be a quasi-convex set. Then $Q^{\triangleright} \subseteq B^{\triangleright}$ and hence $Q=\operatorname{qc}(Q)=$ $\left(Q^{\triangleright}\right)^{\triangleleft} \supseteq\left(B^{\triangleright}\right)^{\triangleleft}$, which implies that $\left(B^{\triangleright}\right)^{\triangleleft}$ is the smallest quasi-convex set which contains $B$.

An abelian topological group is called locally quasi-convex if there is a neighbourhood basis of 0 consisting of quasi-convex sets. It is easy to see 
that the characters of a Hausdorff locally quasi-convex group $G$ separate points and hence $G^{+}$is a Hausdorff group.

Proposition 2.3.

(i) Let $\emptyset \neq B \subseteq G$ where $G$ is an abelian Hausdorff group. Then $B^{\triangleright}=$ $(\mathrm{qc}(B))^{\triangleright}$.

(ii) In a locally quasi-convex Hausdorff group, the quasi-convex hull of a precompact set is precompact.

Proof. (i) Since $B \subseteq \mathrm{qc}(B)$, we obtain $B^{\triangleright} \supseteq(\mathrm{qc}(B))^{\triangleright}$. Conversely, fix $\chi \in B^{\triangleright}$ and $x \in \mathrm{qc}(B)$. Since qc $(B)=\left(B^{\triangleright}\right)^{\triangleleft}$ by $(1)$, we obtain $\chi(x) \in \mathbb{T}_{+}$, and hence the opposite inclusion also holds.

(ii) is (7.12) in [1].

Notation 2.4. For a symmetric subset $A$ of an abelian group $G$ we define $(1 / n) A=\{x \in G: k x \in A \forall 1 \leq k \leq n\}$.

If $U$ is a symmetric neighbourhood of 0 in an abelian topological group then $(1 / n) U$ is also a neighbourhood of 0 .

It is easy to see that $(1 / n) \mathbb{T}_{+}=[-1 / 4 n, 1 / 4 n]$; for a quasi-convex set $A$, we have

$$
(1 / n) A=\bigcap_{\chi \in A^{\triangleright}} \chi^{-1}([-1 / 4 n, 1 / 4 n]), \quad A_{\infty}:=\bigcap_{n \in \mathbb{N}}(1 / n) A=\bigcap_{\chi \in A^{\triangleright}} \operatorname{ker}(\chi) .
$$

The second equality follows from the first one. We have $x \in(1 / n) A$ $\Leftrightarrow \forall 1 \leq k \leq n \quad k x \in A \Leftrightarrow \forall 1 \leq k \leq n, \forall \chi \in A^{\triangleright} \quad k \chi(x)=\chi(k x) \in \mathbb{T}_{+} \Leftrightarrow$ $\forall \chi \in A^{\triangleright} \quad \chi(x) \in(1 / n) \mathbb{T}_{+} \Leftrightarrow \forall \chi \in A^{\triangleright} \quad \chi(x) \in[-1 / 4 n, 1 / 4 n]$.

Proposition 2.5. For a quasi-convex set $A$ of an abelian topological group $G$ and all $n \in \mathbb{N}$ we have:

(i) $(1 / n) A+. . .+(1 / n) A \subseteq A$ and $(1 / 2 n) A+. . .+(1 / 2 n) A \subseteq(1 / 2) A$,

(ii) $(1 / 2 n) A+(1 / 2 n) A \subseteq(1 / n) A$,

(iii) $(1 / n) A+A_{\infty}=(1 / n) A$.

Proof. (i) Let us fix $x_{1}, \ldots, x_{n} \in(1 / n) A$ and $\chi \in A^{\triangleright}$. Since, by (2), $\chi\left(x_{j}\right) \in[-1 / 4 n, 1 / 4 n]$ for all $1 \leq j \leq n$, we obtain $\chi\left(x_{1}+\ldots+x_{n}\right) \in \mathbb{T}_{+}$, and since $\chi \in A^{\triangleright}$ was arbitrary, $x_{1}+\ldots+x_{n} \in A$.

Analogously, the other assertions follow from (2).

COROllary 2.6. Let $A$ be an algebraically quasi-convex subset of an abelian group $G$. Then the sets $((1 / n) A)_{n \in \mathbb{N}}$ form a neighbourhood basis of a (not necessarily Hausdorff) locally quasi-convex group topology on $G$, which will be denoted by $\mathcal{T}_{A}$.

Let $\pi: G \rightarrow G / A_{\infty}$. Then $(1 / n) \pi(A)=\pi((1 / n) A)$ and hence the sets $((1 / n) \pi(A))$ form a neighbourhood basis of a locally quasi-convex Hausdorff group topology on the quotient group, which will be denoted by $\mathcal{T}_{\pi(A)}$. 
Proof. 2.5(ii) implies that the family $((1 / n) A)_{n \in \mathbb{N}}$ has the properties of a neighbourhood basis of 0 of a group topology. Since $A$ is algebraically quasi-convex, (2) implies $(1 / n) A=\bigcap_{\chi \in A^{\triangleright}} \chi^{-1}([-1 / 4 n, 1 / 4 n])$, where $A^{\triangleright}=$ $\left\{\chi \in \operatorname{Hom}(G, \mathbb{T}): \chi(A) \subseteq \mathbb{T}_{+}\right\}$and $\operatorname{Hom}(G, \mathbb{T})$ denotes the set of all homomorphisms $G \rightarrow \mathbb{T}$. Since every character $\chi \in A^{\triangleright}$ is continuous with respect to $\left(G, \mathcal{T}_{A}\right)$, as a consequence of $2.2($ ii) the neighbourhoods $(1 / n) A$ are quasi-convex subsets of $\left(G, \mathcal{T}_{A}\right)$ for all $n \in \mathbb{N}$.

Further,

$$
\begin{aligned}
(1 / n) \pi(A) & =\{\pi(x): k \pi(x) \in \pi(A) \forall 1 \leq k \leq n\} \\
& =\{\pi(x): k x \in \underbrace{A+A_{\infty}}_{=A \text { by } 2.5(\text { iii })} \forall 1 \leq k \leq n\}=\pi((1 / n) A) .
\end{aligned}
$$

Combining this equality with $2.5(\mathrm{ii})$, we find that $((1 / n) \pi(A))_{n \in \mathbb{N}}$ is a neighbourhood basis of a group topology on $G / A_{\infty}$. It is a Hausdorff topology, since $\bigcap_{n \in \mathbb{N}}(1 / n) \pi(A)=\bigcap_{n \in \mathbb{N}} \pi((1 / n) A)=\pi\left(A_{\infty}\right)=\{0\}$.

It is an easy consequence of 2.5 (iii) that the sets $(1 / n) \pi(A)$ are quasiconvex in $\left(G / A_{\infty}, \mathcal{T}_{\pi(A)}\right)$ for every $n \in \mathbb{N}$.

Proposition 2.7. Let $A$ and $B$ be symmetric subsets of an abelian Hausdorff group $G$ and suppose that $0 \in A \cap B$. Then:

(i) $(A+\stackrel{n}{.}+A)^{\triangleright}=\left\{\chi \in G^{\wedge}: \chi(A) \subseteq[-1 / 4 n, 1 / 4 n]\right\}$.

(ii) $\mathrm{qc}(A)+\mathrm{qc}(A) \subseteq \mathrm{qc}(A+A)$.

(iii) $(A+B)^{\triangleright} \supseteq(A+A)^{\triangleright} \cap(B+B)^{\triangleright}$.

(iv) $A^{\triangleright}+A^{\triangleright} \subseteq((1 / 2) A)^{\triangleright}$.

(v) $\left((1 / n) A^{\triangleright}\right)^{\triangleleft}=\mathrm{qc}(A+\stackrel{n}{.}+A)$.

Proof. (i) Fix $\chi \in(A+\stackrel{n}{.}+A)^{\triangleright}$. Since for arbitrary $x \in A$ we have $\chi(k x) \in \mathbb{T}_{+}$for $1 \leq k \leq n$, it follows that $\chi(A) \subseteq[-1 / 4 n, 1 / 4 n]$. Conversely, if $\chi(A) \subseteq[-1 / 4 n, 1 / 4 n]$ then $\chi\left(x_{1}+\ldots+x_{n}\right) \in \mathbb{T}_{+}$for all $x_{1}, \ldots, x_{n} \in A$.

(ii) Let $\chi \in(A+A)^{\triangleright}$. According to (i), $\chi(A) \subseteq[-1 / 8,1 / 8]$ and hence $A \subseteq \chi^{-1}([-1 / 8,1 / 8])$, and since $[-1 / 8,1 / 8]$ is quasi-convex, 2.2(ii) implies that $\mathrm{qc}(A) \subseteq \chi^{-1}([-1 / 8,1 / 8])$. For $x_{1}, x_{2} \in \mathrm{qc}(A)$ we obtain $\chi\left(x_{1}+x_{2}\right) \in \mathbb{T}_{+}$ and hence $x_{1}+x_{2} \in\left((A+A)^{\triangleright}\right)^{\triangleleft}=\mathrm{qc}(A+A)$ by $(1)$.

(iii) Let $\chi \in(A+A)^{\triangleright} \cap(B+B)^{\triangleright}$. According to (i), $\chi(A) \subseteq[-1 / 8,1 / 8]$ and $\chi(B) \subseteq[-1 / 8,1 / 8]$. Hence $\chi(a+b) \in \mathbb{T}_{+}$for all $a \in A$ and $b \in B$.

(iv) Fix $\chi_{1}, \chi_{2} \in A^{\triangleright}$ and $x \in(1 / 2) A$. Since $\chi_{j}((1 / 2) A) \subseteq[-1 / 8,1 / 8]$ $(j \in\{1,2\})$ by $(2)$, we obtain $\left(\chi_{1}+\chi_{2}\right)(x) \in \mathbb{T}_{+}$.

(v) We have $\mathrm{qc}(A+\stackrel{n}{\cdot}+A)=\left(\left(A+\stackrel{n}{ }+{ }^{\circ}+A\right)^{\triangleright}\right)^{\triangleleft}$ by $(1)$. Hence it is sufficient to show that $(A+\stackrel{n}{.}+A)^{\triangleright}=(1 / n) A^{\triangleright}$.

According to (i), $(A+\stackrel{n}{.}+A)^{\triangleright}=\left\{\chi \in G^{\wedge}: \chi(A) \subseteq[-1 / 4 n, 1 / 4 n]\right\}$ and it is easy to prove that this set equals $(1 / n) A^{\triangleright}$. This completes the proof. 
2.3. Schwartz groups. The class of Schwartz groups has recently been introduced in [3]. It contains all Schwartz vector spaces and all nuclear groups, moreover all free locally convex vector spaces $L(X)$ and all free abelian groups $A(X)$ if $X$ is a hemicompact $k$-space. Subgroups, Hausdorff quotients and products of Schwartz groups are again Schwartz groups (3.6 in $[3])$.

The advantage over nuclear groups is that the definition is quite easy:

Definition 2.8. An abelian Hausdorff group $G$ is called a Schwartz group if for every neighbourhood $U$ of 0 there exists a neighbourhood $W$ of 0 and a sequence $\left(F_{n}\right)$ of finite subsets such that $W \subseteq(1 / n) U+F_{n}$ for every $n \in \mathbb{N}$.

\subsection{Character groups}

Lemma 2.9. Let $(G, \tau)$ be an abelian Hausdorff group. For any neighbourhood $U$ of 0 the polar $U^{\triangleright}$ is a compact subset of $G^{\wedge}$ endowed with the compact-open topology.

Proof. This is a consequence of the Arzelà-Ascoli theorem.

Proposition 2.10. Let $(G, \tau)$ be an abelian metrizable group. Then:

(i) Every compact subset of $G^{\wedge}$ is equicontinuous.

(ii) $G^{\wedge}$ is a hemicompact $k$-space (i.e. it has a countable cobasis for the compact sets and a subset is closed iff the intersection with every compact subset is compact).

(iii) $G^{\wedge}$ is a locally quasi-convex Schwartz group.

Proof. (i) Since a metrizable space is a $k$-space, this is a consequence of the Arzelà-Ascoli theorem.

(ii) is Theorem 1 in [8] or (4.7) in [1].

(iii) In (5.6) in [3] it is proved that $G^{\wedge}$ is a Schwartz group if $G$ is metrizable. According to 2.2 (iii), $G^{\wedge}$ is locally quasi-convex.

Proposition 2.11. Let $(G, \tau)$ be an abelian metrizable group. If $G$ is locally quasi-convex, then $G^{\wedge}$ is a nuclear group if and only if $G$ itself is nuclear.

Proof. If $G$ is a nuclear metrizable group then $G^{\wedge}$ is a nuclear group ((16.1) in [4] or (20.35) and (20.36) in [1]). Assume now that $G$ is a metrizable locally quasi-convex group and that $G^{\wedge}$ is nuclear. Since $G$ is metrizable and locally quasi-convex, the canonical mapping $\alpha_{G}: G \rightarrow G^{\wedge \wedge}, x \mapsto(\chi \mapsto$ $\chi(x)$ ), is an embedding (2.10(i) and (6.10) in [1]) and $G^{\wedge}$ is a hemicompact $k$-space (2.10(ii)). So Theorem (6.10) in [2] implies that $G^{\wedge \wedge}$ is a metrizable nuclear group. Since $\alpha_{G}$ is an embedding and subgroups of nuclear groups are nuclear, $G$ must be a nuclear group. 
REMARK 2.12. The character groups of metrizable locally quasi-convex non-nuclear groups provide examples of locally quasi-convex Schwartz groups which are not nuclear.

3. Precompact subsets in locally quasi-convex groups. In this section we intend to prove that the definition of a precompact set in a locally quasi-convex group can be weakened:

Recall that a subset $S$ of a topological group $G$ is precompact if for every 0-neighbourhood $U$ the set $S$ is covered by a finite number of translates of $U$, i.e. there exists a finite set $F \subseteq G$ (or equivalently, $F \subseteq S$ ) such that $S \subseteq F+U$.

The weaker version (which in the case of quasi-convex groups will be shown to be equivalent to precompactness) and which we will call qc-precompactness, is: for every neighbourhood $U$ of 0 there exists a finite set $F \subseteq G$ such that

$$
S \subseteq \mathrm{qc}(F+U) .
$$

Our aim is to show that every qc-precompact set in a locally quasi-convex group is precompact.

First we need a lemma which will ensure a completeness property:

LEMmA 3.1. Let $\mathcal{O}_{1}$ and $\mathcal{O}_{2}$ be Hausdorff group topologies on an abelian group $G$ and assume that id : $\left(G, \mathcal{O}_{1}\right) \rightarrow\left(G, \mathcal{O}_{2}\right)$ is continuous. Assume moreover that there exists a 0 -neighbourhood basis in $\left(G, \mathcal{O}_{1}\right)$ consisting of $\mathcal{O}_{2}$-closed sets. If $K \subseteq G$ is complete for $\mathcal{O}_{2}$, then it is also complete for $\mathcal{O}_{1}$.

Proof. This is Theorem 3.2.4 in [21]. Only the group, but not the vector space structure is used in the proof.

LEMMa 3.2. Let $\mathcal{M}$ be a family of subsets of an abelian topological group $G$ which has the following properties:

(a) $\bigcup_{M \in \mathcal{M}} M=G$,

(b) $\mathcal{M}$ is closed with respect to finite unions,

(c) $\mathcal{M}$ is closed with respect to finite sums.

Then the family of polars $\left(M^{\triangleright}\right)_{M \in \mathcal{M}}$ forms a 0-neighbourhood basis $\mathcal{V}$ for a Hausdorff group topology $\tau_{\mathcal{M}}$ on $G^{\wedge}$, called the topology of uniform convergence on elements of $\mathcal{M}$.

Proof. (a) implies that $\bigcap M^{\triangleright}=\{0\}$. It is a consequence of (b) that $M_{1}^{\triangleright} \cap M_{2}^{\triangleright}=\left(M_{1} \cup M_{2}\right)^{\triangleright} \in \mathcal{V}$ for all $M_{1}, M_{2} \in \mathcal{M}$. Fix $M \in \mathcal{M}$. Because of (a) and (b) we may assume that $0 \in M$. Since $M \subseteq(1 / 2)(M+M), 2.7$ (iv) implies $(M+M)^{\triangleright}+(M+M)^{\triangleright} \subseteq((1 / 2)(M+M))^{\triangleright} \subseteq M^{\triangleright}$. Hence $\mathcal{V}$ induces a Hausdorff group topology on $G^{\wedge}$. 
Example 3.3. The families $\mathcal{F}$ of finite sets, $\mathcal{C}$ of compact sets, and $\mathcal{P}$ of precompact sets have the properties stated above and hence induce the topologies $\tau_{\mathcal{F}}, \tau_{\mathcal{C}}$, and $\tau_{\mathcal{P}}$.

Notation 3.4. In a locally quasi-convex group $G$ we consider the set $\mathcal{S}$ of all qc-precompact subsets.

LEMma 3.5. The family $\mathcal{S}$ has the properties stated in 3.2 .

Proof. Since all singletons belong to $\mathcal{S}$, (a) is obviously satisfied. Let $S_{1}, S_{2} \in \mathcal{S}$. We fix a neighbourhood $U$ of 0 and choose a neighbourhood $W$ of 0 which satisfies $W+W \subseteq U$. By assumption, there exists a finite set $F$ such that $S_{i} \subseteq \mathrm{qc}(F+W)(i \in\{1,2\})$. Hence $S_{1} \cup S_{2} \subseteq \mathrm{qc}(F+W)$ and $S_{1}+S_{2} \subseteq \mathrm{qc}(F+W)+\mathrm{qc}(F+W) \stackrel{2.7(\mathrm{ii})}{\subseteq} \mathrm{qc}(F+F+W+W) \subseteq \mathrm{qc}(F+F+U)$.

Proposition 3.6. Let $(G, \tau)$ be a metrizable locally quasi-convex group. Let $\tau_{\mathcal{S}}$ be the topology of uniform convergence on members of $\mathcal{S}$.

(i) $\tau_{\mathcal{S}}$ coincides with the compact-open topology.

(ii) Every qc-precompact set of $(G, \tau)$ is precompact.

Proof. Since $\mathcal{S} \supseteq \mathcal{P} \supseteq \mathcal{C} \supseteq \mathcal{F}$, the identity mappings

$$
\left(G^{\wedge}, \tau_{\mathcal{S}}\right) \rightarrow\left(G^{\wedge}, \tau_{\mathcal{P}}\right) \rightarrow\left(G^{\wedge}, \tau_{\mathcal{C}}\right) \rightarrow\left(G^{\wedge}, \tau_{\mathcal{F}}\right)
$$

are continuous.

(i) We want to show that the first three topologies coincide. First we shall show that every $\tau_{\mathcal{C}}$-compact set $K$ is also compact with respect to $\tau_{\mathcal{S}}$. Because of 2.10(i) and 2.9, we may assume that $K=U^{\triangleright}$ for some $U \in \mathcal{U}(0)$.

First we shall show that $U^{\triangleright}$ is precompact in $\left(G^{\wedge}, \tau_{\mathcal{S}}\right)$. Therefore, we fix $S \in \mathcal{S}$. Let $W \in \mathcal{U}(0)$ be quasi-convex such that $W+W \subseteq(1 / 2) U$. By assumption, there exists a symmetric and finite set $F$ with $0 \in F$ such that $S \subseteq \mathrm{qc}(F+W)$. This implies

$$
S^{\triangleright} \supseteq(\mathrm{qc}(F+W))^{\triangleright} \stackrel{2.3(\mathrm{i})}{=}(F+W)^{\triangleright} \stackrel{2.7(\text { iii })}{\supseteq}(F+F)^{\triangleright} \cap(W+W)^{\triangleright} .
$$

By the continuity of $\left(G^{\wedge}, \tau_{\mathcal{C}}\right) \rightarrow\left(G^{\wedge}, \tau_{\mathcal{F}}\right)$, the set $U^{\triangleright}$ is also $\tau_{\mathcal{F}}$-compact, hence there exists a finite set $H \subseteq U^{\triangleright}$ such that $U^{\triangleright} \subseteq H+(F+F)^{\triangleright}$. Since $H \subseteq U^{\triangleright}$, we even have

$$
U^{\triangleright} \subseteq H+\left((F+F)^{\triangleright} \cap\left(U^{\triangleright}+U^{\triangleright}\right)\right) .
$$

Hence we obtain

$$
(F+F)^{\triangleright} \cap\left(U^{\triangleright}+U^{\triangleright}\right) \stackrel{2.7(\text { iv) }}{\subseteq}(F+F)^{\triangleright} \cap((1 / 2) U)^{\triangleright} \subseteq(F+F)^{\triangleright} \cap(W+W)^{\triangleright} \stackrel{(3)}{\subseteq} S^{\triangleright},
$$

which enables us to reformulate (4):

$$
U^{\triangleright} \subseteq H+S^{\triangleright} .
$$

This shows that $U^{\triangleright}$ is precompact in $\tau_{\mathcal{S}}$. 
$\left(U^{\triangleright},\left.\tau_{\mathcal{S}}\right|_{U^{\triangleright}}\right)$ is complete according to 3.1: $S^{\triangleright}$ is closed in $\tau_{\mathcal{F}}$ and hence in $\tau_{\mathcal{C}}$, and $\left(U^{\triangleright},\left.\tau_{\mathcal{C}}\right|_{U^{\triangleright}}\right)$ is compact $(2.9)$, therefore complete.

Up to now we have shown that every $\tau_{\mathcal{C}}$-compact set is also compact with respect to $\tau_{\mathcal{S}}$.

According to $2.10(\mathrm{ii}),\left(G^{\wedge}, \tau_{\mathcal{C}}\right)$ is a $k$-space. In order to show that $\left(G^{\wedge}, \tau_{\mathcal{C}}\right)$ $\rightarrow\left(G, \tau_{\mathcal{S}}\right)$ is continuous, it is sufficient to verify that on every subset $K$ of $G^{\wedge}$ which is compact with respect to $\tau_{\mathcal{C}}$ both topologies coincide. Since we know that $K$ is also compact with respect to $\tau_{\mathcal{S}}$, this indeed holds true (because $\left.\tau_{\mathcal{C}}\right|_{K}$ cannot be strictly coarser that $\left.\tau_{\mathcal{S}}\right|_{K}$ ). This proves (i).

(ii) For every $S \in \mathcal{S}$, there exists a compact subset $C \subseteq G$ such that $S^{\triangleright} \supseteq C^{\triangleright}$, which implies qc $(S) \subseteq \mathrm{qc}(C)$. Since qc $(C)$ is precompact (2.3(ii)), the assertion follows.

THEOREM 3.7. In a locally quasi-convex group $(G, \mathcal{O})$ all qc-precompact sets are precompact.

Proof. Fix $S \in \mathcal{S}$. Let $W$ be a quasi-convex neighbourhood of 0 in $G$ and let $\pi:(G, \mathcal{O}) \rightarrow\left(G / W_{\infty}, \mathcal{T}_{\pi(W)}\right)$ be the canonical projection. 2.6 implies $\pi^{-1}((1 / n) \pi(W))=\pi^{-1}(\pi((1 / n) W))=(1 / n) W+W_{\infty}=(1 / n) W$ by 2.5 (iii), which implies that $\pi$ is continuous. By assumption, for every $n \in \mathbb{N}$, there exists a finite set $F_{n} \subseteq G$ such that $S \subseteq \mathrm{qc}\left(F_{n}+(1 / n) W\right)$. We have

$$
\begin{aligned}
\mathrm{qc}\left(F_{n}+(1 / n) W\right) & \stackrel{2.2(\mathrm{ii})}{\subseteq} \pi^{-1}\left(\overline{\mathrm{qc}}\left(\pi\left(F_{n}+(1 / n) W\right)\right)\right) \\
& \stackrel{2.6}{=} \pi^{-1}\left(\overline{\mathrm{qc}}\left(\pi\left(F_{n}\right)+(1 / n) \pi(W)\right)\right)
\end{aligned}
$$

where $\overline{\mathrm{qc}}$ denotes the quasi-convex hull in the quotient group $\left(G / W_{\infty}, \mathcal{T}_{\pi(W)}\right)$. From this we derive

$$
\pi(S) \subseteq \pi\left(\mathrm{qc}\left(F_{n}+(1 / n) W\right)\right) \subseteq \overline{\mathrm{qc}}\left(\pi\left(F_{n}\right)+(1 / n) \pi(W)\right) .
$$

Since the sets $((1 / n) \pi(W))$ form a neighbourhood basis of 0 in the locally quasi-convex quotient group $\left(G / W_{\infty}, \mathcal{I}_{\pi(W)}\right)$, the set $\pi(S)$ is qc-precompact (more precisely: $\overline{\text { qc}}$-precompact). 3.6 implies that $\pi(S)$ is precompact with respect to $\mathcal{T}_{\pi(W)}$. As a consequence, there exists a finite set $F \subseteq G$ with $\pi(S) \subseteq \pi(F)+\pi(W)$ and hence $S \subseteq F+W+W_{\infty}=F+W$ by 2.5 (iii). Since $W$ was arbitrary, we have shown that $S$ is precompact.

EXAMPLE 3.8. Let $G$ be an abelian topological group which has only the trivial continuous character. Then $G$ is qc-precompact, since $G=\mathrm{qc}(\{0\})$, but it is not precompact, because the characters would then separate points.

Assume that the continuous characters of the abelian topological group $(G, \tau)$ separate points. The family of sets $(\mathrm{qc}(U))_{U \in \mathcal{U}(0)}$ forms a neighbourhood basis of a Hausdorff locally quasi-convex group topology $\tau_{\mathrm{qc}}$ on $G$ (see [7] or (6.18) in [1] for details). 
COROLlary 3.9. Suppose that the continuous characters of the abelian group $(G, \tau)$ separate points. Then the qc-precompact sets in $(G, \tau)$ coincide with the precompact sets in $\left(G, \tau_{\mathrm{qc}}\right)$.

Proof. Let $S \subseteq G$ be qc-precompact in $(G, \tau)$. Fix a neighbourhood $U \in$ $\mathcal{U}_{\tau}(G)$. There exists a finite set $F$ such that $S \subseteq \mathrm{qc}(F+U) \subseteq \mathrm{qc}(F+\mathrm{qc}(U))$, which shows that $S$ is qc-precompact in $\left(G, \tau_{\mathrm{qc}}\right)$. Now 3.7 implies that $S$ is precompact in $\left(G, \tau_{\mathrm{qc}}\right)$.

Conversely, assume that $S \subseteq G$ is precompact in $\left(G, \tau_{\text {qc }}\right)$. Fix $U \in \mathcal{U}_{\tau}(0)$ and choose $W \in \mathcal{U}_{\tau}(0)$ such that $\mathrm{qc}(W)+\mathrm{qc}(W) \subseteq \mathrm{qc}(U)$. Since $S$ is precompact in $\left(G, \tau_{\mathrm{qc}}\right)$, there exists a finite set $F$ such that $S \subseteq F+\mathrm{qc}(W)$.

Further, for $\widetilde{F}:=(\{0\} \cup F)+(\{0\} \cup F)$ we obtain

$$
\begin{aligned}
& (\widetilde{F}+U)^{\triangleright} \subseteq \widetilde{F}^{\triangleright} \cap U^{\triangleright} \stackrel{2.3(\mathrm{i})}{=} \widetilde{F}^{\triangleright} \cap(\mathrm{qc}(U))^{\triangleright} \\
& \subseteq((\{0\} \cup F)+(\{0\} \cup F))^{\triangleright} \cap(\mathrm{qc}(W)+\mathrm{qc}(W))^{\triangleright} \stackrel{2.7(\mathrm{ii})}{\subseteq}(F+\mathrm{qc}(W))^{\triangleright} .
\end{aligned}
$$

This implies $S \subseteq F+\mathrm{qc}(W) \subseteq \mathrm{qc}(\widetilde{F}+U)$ and shows that $S$ is qc-precompact in $(G, \tau)$.

EXAMPLE 3.10. Let $(G, \tau)$ be a completely metrizable abelian group such that the characters separate points, but assume that it is not locally quasi-convex, e.g. $\ell^{p}$ with $0<p<1$.

Since both $(G, \tau)$ and $\left(G, \tau_{\mathrm{qc}}\right)$ are metrizable and $\tau$ is strictly finer than $\tau_{\mathrm{qc}}$, there exists a sequence $\left(x_{n}\right)$ tending to 0 in $\tau_{\mathrm{qc}}$ but not in $\tau$. Then $S:=\left\{x_{n}: n \in \mathbb{N}\right\} \cup\{0\}$ is a compact subset of $\left(G, \tau_{\mathrm{qc}}\right)$. According to 3.9 , it is qc-precompact in $(G, \tau)$. We shall show that it is not precompact. Since $(G, \tau) \rightarrow\left(G, \tau_{\mathrm{qc}}\right)$ is continuous, $S$ is closed in $(G, \tau)$ and hence complete. If it were precompact, it would be compact, but then both topologies would coincide on $S$. This contradiction shows that $S$ is not precompact in $(G, \tau)$.

\section{The Glicksberg theorem}

Proposition 4.1. Let $U$ be a neighbourhood of 0 in the abelian topological group $H$ and suppose that $\langle U\rangle=H$. Then

$$
\text { id : }\left(H^{\wedge}, \mathcal{T}_{U^{\triangleright}}\right) \rightarrow\left(H^{\wedge}, \tau_{\mathcal{F}}\right)
$$

is continuous and $\left(H^{\wedge}, \mathcal{T}_{U \triangleright}\right)$ is complete and metrizable. (A neighbourhood basis of this group is given by the sets $(1 / n) U^{\triangleright}$.

Proof. Observe first that $\left(U^{\triangleright}\right)_{\infty}=\left\{\chi \in H^{\wedge}:\langle\chi\rangle \subseteq U^{\triangleright}\right\}=\left\{\chi \in H^{\wedge}\right.$ : $\chi(U)=\{0\}\}=\{0\}$. Hence $\mathcal{T}_{U^{\triangleright}}$ is a Hausdorff topology on $H^{\wedge}$. Since $\mathcal{T}_{U^{\triangleright}}$ is first countable, $\left(H^{\wedge}, \mathcal{T}_{U^{\triangleright}}\right)$ is metrizable.

For fixed $u \in U$ the homomorphism $\alpha_{H}(u): H^{\wedge} \rightarrow \mathbb{T}, \chi \mapsto \chi(u)$, is continuous with respect to $\mathcal{T}_{U^{\triangleright}}$, because $\alpha_{H}(u)\left(U^{\triangleright}\right) \subseteq \mathbb{T}_{+}$. Since we assumed $\langle U\rangle=H$, the continuity of id $:\left(H^{\wedge}, \mathcal{T}_{U^{\triangleright}}\right) \rightarrow\left(H^{\wedge}, \tau_{\mathcal{F}}\right)$ follows. 
In order to show that $\left(H^{\wedge}, \mathcal{T}_{U^{\triangleright}}\right)$ is complete, we fix a Cauchy net $\left(x_{i}\right)$ in $\left(H^{\wedge}, \mathcal{T}_{U \triangleright}\right)$. There exists $i_{0} \in I$ such that $x_{i}-x_{i_{0}} \in U^{\triangleright}$ for all $i \geq i_{0}$. So we may and do assume that $x_{i} \in U^{\triangleright}$ for all $i \in I$. Hence we are in a position to prove that $\left(U^{\triangleright},\left.\mathcal{T}_{U^{\triangleright}}\right|_{U^{\triangleright}}\right)$ is complete. This will follow from 3.1: Observe first that the sets $(1 / n) U^{\triangleright}=\left\{\chi \in H^{\wedge}: \chi(U) \subseteq[-1 / 4 n, 1 / 4 n]\right\}$ are closed in $\left(H^{\wedge}, \tau_{\mathcal{F}}\right)$. Further, the set $U^{\triangleright}$ is compact in $\tau_{\mathcal{C}}(2.9)$ and hence also in $\tau_{\mathcal{F}}$.

In the following proposition we shall show that every weakly compact $S$ set is "qc-bounded", i.e. for every neighbourhood $U \in \mathcal{U}(0)$ there exist a finite set $F$ and a natural number $n$ such that $S \subseteq F+\mathrm{qc}(U+. \stackrel{n}{.}+U)$.

Proposition 4.2. Let $G$ be a locally quasi-convex group and let $S \subseteq G$ be a weakly compact subset. For every $U \in \mathcal{U}(0)$ there exists a weakly compact subset $S_{0} \subseteq\langle U\rangle$ and a finite set $F$ such that $S \subseteq F+S_{0}$. Further, there is a natural number $n \in \mathbb{N}$ such that

$$
S_{0} \subseteq \mathrm{qc}(U+. . n+U) .
$$

Proof. Let $\pi: G \rightarrow G /\langle U\rangle$ be the canonical projection. Since $U$ is a neighbourhood, the quotient $G /\langle U\rangle$ is discrete. Further, $\pi$ is continuous if both groups are endowed with their weak topologies $(2.1)$. So $\pi(S)$ is a weakly compact set in $G /\langle U\rangle$. According to the Glicksberg theorem for discrete groups, $\pi(S)$ is finite. (For an easy proof of the Glicksberg theorem for discrete groups, see [10].)

Hence there exists a finite set $F \subseteq G$ such that $\pi(S)=\pi(F)$. The set $S_{0}:=\langle U\rangle \cap(S-F)$ is weakly compact, contained in $\langle U\rangle$, and satisfies $S \subseteq F+S_{0}$. Therefore, it is sufficient to show that $S_{0} \subseteq \mathrm{qc}(U+\ldots+U)$ for some $n \in \mathbb{N}$. $H:=\langle U\rangle$ is an open and hence dually embedded subgroup of $(G, \tau) .2 .1$ (ii) implies that $S_{0}$ is a weakly compact subset of $H$.

Consider the mapping

$$
m: S_{0} \times H^{\wedge} \rightarrow \mathbb{T}, \quad(x, \chi) \mapsto \chi(x),
$$

where $S_{0}$ is endowed with the topology coming from $H^{+}$and $H^{\wedge}$ is endowed with the topology $\mathcal{T}_{U^{\triangleright}}$. The continuity of $m$ in the first coordinate (when the second is fixed) holds by the definition of the weak topology. The continuity in the second variable is a consequence of 4.1. But $S_{0}$ is compact and $\left(H^{\wedge}, \mathcal{T}_{U^{\triangleright}}\right)$ is completely metrizable (4.1), which permits us to apply Namioka's theorem (Theorem 1.2 in [24]). Hence there exists a dense $G_{\delta}$-subset $Y$ of $H^{\wedge}$ such that $m$ is continuous at every point $(x, \chi) \in S_{0} \times Y$. We fix $\chi \in Y$. Since $S_{0}$ is compact, there exists $n \in \mathbb{N}$ such that $m\left(x, \chi^{\prime}\right)-m(x, \chi) \in \mathbb{T}_{+}$for all $x \in S_{0}$ and all $\chi^{\prime} \in \chi+(1 / n) U^{\triangleright}$. This implies $\psi(x) \in \mathbb{T}_{+}$for all $x \in S_{0}$ and $\psi \in(1 / n) U^{\triangleright}$, or equivalently, $S_{0} \subseteq\left((1 / n) U^{\triangleright}\right)^{\triangleleft}$. It remains to observe that $\left((1 / n) U^{\triangleright}\right)^{\triangleleft}=\mathrm{qc}(U+. . .+U)$ by $2.7(\mathrm{v})$. 
REMARK 4.3. It is a well known fact in the theory of locally convex vector spaces that every weakly bounded set is bounded.

Indeed, fix a weakly bounded subset $S$ of a locally convex vector space $V$ and an absolutely convex neighbourhood $U$ of 0 . Every $x \in S$ induces a continuous linear function $T_{x}:\left(V, \mathcal{T}_{U}\right)^{\prime} \rightarrow \mathbb{R}, \phi \mapsto \phi(x)$. By assumption, this family is pointwise bounded and since $\left(V, \mathcal{T}_{U}\right)^{\prime}$ is a Banach space, the uniform, boundedness principle implies that $\left(T_{x}\right)$ is uniformly bounded, which means that $\left|T_{x}(\phi)\right|=|\phi(x)| \leq c$ for a suitable constant $c>0$ and all $\phi \in U^{\circ}$, the polar of $U$, and all $x \in U$. An equivalent formulation is that

$$
S \times\left(V, \mathcal{T}_{U}\right)^{\prime} \rightarrow \mathbb{R}, \quad(x, \phi) \mapsto \phi(x),
$$

is jointly continuous at every point $(x, 0)(x \in S)$.

This was generalized in the preceding proof for topological groups where the uniform boundedness principle was replaced by Namioka's theorem on joint continuity.

TheOREM 4.4. Let $(G, \tau)$ be a locally quasi-convex Schwartz group. Then every weakly compact set is compact.

Proof. We fix a weakly compact set $S \subseteq G$.

First we want to show that $S$ is precompact. Therefore, we fix a quasiconvex neighbourhood $W$ of 0 . Since $G$ is a Schwartz group, there exist a quasi-convex neighbourhood $U$ of 0 and a sequence $\left(F_{n}\right)$ of finite subsets such that

$$
U \subseteq F_{n}+(1 / 2 n) W \quad \text { for all } n \in \mathbb{N},
$$

in particular,

$$
\begin{aligned}
& U+\stackrel{m}{?}+U \subseteq F_{m}+(1 / 2 m) W+\stackrel{m}{.}+F_{m}+(1 / 2 m) W \\
& \stackrel{2.5(\mathrm{i})}{\subseteq} \widetilde{F}_{m}+(1 / 2) W
\end{aligned}
$$

for $\widetilde{F}_{m}=F_{m}+\stackrel{m}{.}+F_{m}$ and all $m \in \mathbb{N}$.

According to 4.2 , there is a finite set $F$ and a weakly compact set $S_{0} \subseteq\langle U\rangle$ such that $S \subseteq F+S_{0}$; further, there is a natural number $m \in \mathbb{N}$ such that

$$
S_{0} \subseteq \mathrm{qc}(U+\stackrel{m}{.}+U) \stackrel{(5)}{\subseteq} \mathrm{qc}\left(\widetilde{F}_{m}+(1 / 2) W\right) .
$$

This implies

$$
\begin{aligned}
S \subseteq F+S_{0} & \stackrel{(6)}{\subseteq} F+\mathrm{qc}\left(\widetilde{F}_{m}+(1 / 2) W\right) \\
& \subseteq \mathrm{qc}\left(\left(F \cup \widetilde{F}_{m}\right)+(1 / 2) W\right)+\mathrm{qc}\left(\left(F \cup \widetilde{F}_{m}\right)+(1 / 2) W\right) \\
& 2.7(\mathrm{ii}) \\
& \subseteq \mathrm{qc}\left(\left(F \cup \widetilde{F}_{m}\right)+(1 / 2) W+\left(F \cup \widetilde{F}_{m}\right)+(1 / 2) W\right) \\
& \subseteq \mathrm{qc}\left(\left(F \cup \widetilde{F}_{m}\right)+\left(F \cup \widetilde{F}_{m}\right)+W\right) .
\end{aligned}
$$


Since $W$ was arbitrary, this shows that $S$ is qc-precompact, and 3.7 implies that it is precompact.

It remains to show that $S$ is complete in $(G, \tau)$. Consider the identity $(G, \tau) \rightarrow G^{+}$, which is obviously continuous. $(G, \tau)$ is locally quasi-convex and each quasi-convex set is weakly closed; further, $S$ is weakly compact and hence weakly complete, so 3.1 implies that $\left(S,\left.\tau\right|_{S}\right)$ is complete. The assertion follows.

Comfort, Trigos-Arrieta and $\mathrm{Wu}$ introduced the following

Definition 4.5 ([11]). A MAP $G$ is said to strongly respect compactness if for every closed, metrizable subgroup $N \leq b G$ the following holds: if $A \subseteq G$ is such that $\phi(A)$ is compact (where $\phi: b G \rightarrow b G / N$ is the canonical projection) then $A \cdot(N \cap G)$ is compact.

For the special case $N=\{0\}$, the condition reduces to respecting compactness and hence every group which strongly respects compactness respects compactness.

It was shown in [11] that every LCA groups strongly respects compactness, and in [14] that for every Dieudonné complete space $X$ the free abelian group $A(X)$ strongly respects compactness.

So it is natural to ask:

Question 4.6. Does every locally quasi-convex Schwartz group strongly respect compactness?

Further, it was shown in [6] that every nuclear group respects countable compactness and pseudocompactness. If, in addition, the group is complete, then it respects functional boundedness.

QUESTION 4.7. Do analogous results hold true for locally quasi-convex Schwartz groups?

5. An application. Finally, we are going to apply the Glicksberg theorem for Schwartz groups to character groups of metrizable groups.

Proposition 5.1. Let $G$ be a metrizable abelian group. Every weakly compact subset of $G^{\wedge}$ is equicontinuous.

Proof. According to 2.10(iii), the character group of $G$ is a locally quasiconvex Schwartz group. Hence 4.4 implies that every weakly compact set is compact with respect to the compact-open topology. Applying 2.10(i), we conclude that it must be equicontinuous.

REMARK 5.2. This result has been proved by other methods in (2.3)(c) of [23].

In (1.5) of [9] it has been shown that every $\sigma\left(G^{\wedge}, G\right)$-compact subset of the character group $G^{\wedge}$ of a complete metrizable group is equicontinuous 
$\left(\sigma\left(G^{\wedge}, G\right)\right.$ is the topology on $G^{\wedge}$ induced by the mapping $G^{\wedge} \rightarrow \mathbb{T}^{G}, \chi \mapsto$ $\left.(\chi(x))_{x \in G}\right)$. This result is stronger, but requires completeness. The next proposition will show that it is not possible to drop this assumption.

Proposition 5.3. A countable locally quasi-convex Hausdorff group $(G, \tau)$ such that every $\sigma\left(G^{\wedge}, G\right)$-compact subset of $G^{\wedge}$ is equicontinuous must be discrete.

Proof. Let $H=G^{\wedge}$ be endowed with the $\sigma\left(G^{\wedge}, G\right)$ topology. Then $H \rightarrow$ $\mathbb{T}^{G}, \chi \mapsto(\chi(x))_{x \in G}$, is an embedding and hence $H$ is a metrizable (since $G$ was assumed to be countable) precompact group. By Theorem 2 in [8] or (4.10) in [1], its character group is discrete. Let us show that $H^{\wedge} \cong$ $G$. Algebraically, this is clear. So let us fix a compact subset $K$ of $H$. By assumption, $K$ is equicontinuous, hence there exists a neighbourhood $U$ of 0 such that $K \subseteq U^{\triangleright}$. We may assume that $K=U^{\triangleright}$, because $U^{\triangleright}$ is $\sigma\left(G^{\wedge}, G\right)$ compact as well. This implies $K^{\triangleright}=\left\{x \in G: \chi(x) \in \mathbb{T}_{+} \forall \chi \in U^{\triangleright}\right\}=\mathrm{qc}(U)$. Since $(G, \tau)$ is locally quasi-convex, $H^{\wedge} \cong G$ and hence $G$ is discrete.

Acknowledgements. I am indebted to Elena Martín-Peinador for helpful discussions, to Xabier Domínguez, Vaja Tarieladze, and the referee for their careful reading of the manuscript, and to Dikran Dikranjan and MaríaJesus Chasco who bore in remembrance the question underlying this paper.

\section{References}

[1] L. Außenhofer, Contributions to the duality theory of abelian topological groups and to the theory of nuclear groups, Dissertationes Math. 384 (1999).

[2] - On the nuclearity of dual groups, preprint, 2007.

[3] L. Außenhofer, M. J. Chasco, X. Domínguez and V. Tarieladze, On Schwartz groups, Studia Math. 181 (2007), 199-210.

[4] W. Banaszczyk, Additive Subgroups of Topological Vector Spaces, Lecture Notes in Math. 1466, Springer, Berlin, 1991.

[5] W. Banaszczyk and E. Martín-Peinador, The Glicksberg theorem on weakly compact sets for nuclear groups, in: E. Coplakova et al. (eds.), Papers on General Topology and Applications (Amsterdam, 1994), Ann. New York Acad. Sci. 788, New York Academy of Sciences, 1996, 34-39.

[6] W. Banaszczyk and E. Martín-Peinador, Weakly pseudocompact subsets of nuclear groups, J. Pure Appl. Algebra 138 (1999), 99-106.

[7] M. Bruguera, Some properties of locally quasi-convex groups, Topology Appl. 77 (1997), 87-94.

[8] M. J. Chasco, Pontryagin duality for metrizable groups, Arch. Math. (Basel) 70 (1998), 22-28.

[9] M. J. Chasco, E. Martín-Peinador and V. Tarieladze, On Mackey topology for groups, Studia Math. 132 (1999), 257-284.

[10] W. W. Comfort and F. J. Trigos-Arrieta, Remarks on a theorem of Glicksberg, in: General Topology and Applications (New York, 1989), Lecture Notes Pure Appl. Math. 134, Dekker, 1991, 25-33. 
[11] W. W. Comfort, F. J. Trigos-Arrieta and T.-S. Wu, The Bohr compactification, modulo a metrizable subgroup, Fund. Math. 143 (1993), 119-136.

[12] - , - - Correction to the paper "The Bohr compactification, modulo a metrizable subgroup", ibid. 152 (1997), 97-98.

[13] H. H. Corson and I. Glicksberg, Compactness in $\operatorname{Hom}(G, H)$, Canad. J. Math. 22 (1970), 164-170.

[14] J. Galindo and S. Hernández, The concept of boundedness and the Bohr compactification of a MAP abelian group, Fund. Math. 159 (1999), 195-218.

[15] - - - Interpolation sets and the Bohr topology of locally compact groups, Adv. Math. 188 (2004), 51-68.

[16] I. Glicksberg, Uniform boundedness for groups, Canad. J. Math. 14 (1962), 269-276.

[17] J. Hejcman, Boundedness in uniform spaces and topological groups, Czechoslovak Math. J. 9 (84) (1959), 544-562.

[18] S. Hernández, The dimension of an LCA group in its Bohr topology, Topology Appl. 86 (1998), 63-67.

[19] S. Hernández, J. Galindo and S. Macario, A characterization of the Schur property by means of the Bohr topology, ibid. 97 (1999), 99-108.

[20] S. Hernández and S. Macario, Invariance of compactness for the Bohr topology, ibid. 111 (2001), 161-173.

[21] H. Jarchow, Locally Convex Spaces, Mathematische Leitfäden, B. G. Teubner, Stuttgart, 1981.

[22] H. Leptin, Abelsche Gruppen mit kompakten Charaktergruppen und Dualitätstheorie gewisser linear topologischer abelscher Gruppen, Abh. Math. Sem. Univ. Hamburg 19 (1955), 244-263.

[23] E. Martín-Peinador and V. Tarieladze, A property of Dunford-Pettis type in topological groups, Proc. Amer. Math. Soc. 132 (2004), 1827-1837.

[24] I. Namioka, Separate continuity and joint continuity, Pacific J. Math. 51 (1974), $515-531$.

[25] D. Remus and F. J. Trigos-Arrieta, Abelian groups which satisfy Pontryagin duality need not respect compactness, Proc. Amer. Math. Soc. 117 (1993), 1195-1200.

[26] —, - , The Bohr topology of Moore groups, Topology Appl. 97 (1999), 85-98.

[27] F. J. Trigos-Arrieta, Continuity, boundedness, connectedness and the Lindelöf property for topological groups, J. Pure Appl. Algebra 70 (1991), 199-210.

[28] —, Pseudocompactness on groups, in: General Topology and Applications (New York, 1989), Lecture Notes Pure Appl. Math. 134, Dekker, 1991, 369-378.

[29] J. P. Troallic, Boundedness in $\mathcal{C}_{p}(X, Y)$ and equicontinuity, Topology Appl. 108 (2000), 79-89.

[30] T.-S. Wu and L. Riggins, Maximally almost periodic groups and a theorem of Glicksberg, in: S. Andima et al. (eds.), Papers on General Topology and Applications (Gorham, ME, 1995), Ann. New York Acad. Sci. 806, New York Academy of Sciences, 1996, 454-464.

Mathematisch-Geographische Fakultät

Universität Eichstätt-Ingolstadt

Ostenstr. 26

D-85072 Eichstätt, Germany

E-mail: lydia.aussenhofer@ku-eichstaett.de 